\title{
INTEGRABILITY OF RECIPROCALS OF THE GREEN'S FUNCTION FOR ELLIPTIC OPERATORS: COUNTEREXAMPLES
}

\author{
M. CRISTINA CERUTTI
}

(Communicated by Barbara L. Keyfitz)

\begin{abstract}
We construct examples of elliptic operators for which the set of points where the reciprocal $1 / g(x, \cdot)$ of the Green's function is not locally integrable in a dense set of points.
\end{abstract}

\section{INTRODUCTION}

This paper deals with Green's functions for second-order linear strongly elliptic operators in $\mathbb{R}^{n}$, in nondivergence form, with bounded measurable coefficients, in a smooth domain $D$. More precisely we will consider elliptic operators of the form

$$
L=\sum_{i, j=1}^{n} a_{i j}(x) D_{i j}^{2}
$$

where $D_{i j}^{2}=\partial^{2} / \partial x_{i} \partial x_{j}$, the functions $a_{i j}$ are defined on some bounded domain $D \subset \mathbb{R}^{n}$ and satisfy the condition $\lambda|\xi|^{2} \leq \sum_{i, j=1}^{n} a_{i j}(x) \xi_{i} \xi_{j} \leq \Lambda|\xi|^{2}$ $\forall x \in D$ and $\forall \xi=\left(\xi_{1}, \ldots, \xi_{n}\right) \in \mathbb{R}^{n}$ for some $0<\lambda \leq \Lambda$, and $a_{i j}(x)=a_{j i}(x)$.

If the coefficients $a_{i j} \in C(\bar{D})$ at least, it is well known that the Dirichlet problem

$$
\begin{cases}L u=-f & \text { in } D \\ u=0 & \text { on } \partial D\end{cases}
$$

with $f \in L^{p}(D)$ for some finite $p>n / 2$ has a unique strong solution $u \in$ $W_{\text {loc }}^{2, p}(D) \cap C(\bar{D})$ (see, e.g., [8]). Also a result of Pucci and Aleksandrov (see $[1,11])$ states that $\sup _{D}|u| \leq C\|f\|_{L^{n}(D)}$. Therefore the functional $f \rightarrow u(x)$, $x \in D$, is a bounded linear functional on $L^{n}(D)$ for each $x$ and by the Riesz representation theorem, there exists a unique Green's function for the operator

Received by the editors August 5, 1991 and, in revised form, January 21, 1992.

1991 Mathematics Subject Classification. Primary 35R05, 35A08, 35J15, 35C15.

Partially supported by the NSF Grant \#DMS 842133-04 and by the Italian C.N.R., G.N.A.F.A. 
$L$ in $D, g(x, \cdot) \in L^{n /(n-1)}(D)$, such that

$$
\begin{gathered}
u(x)=\int_{D} g(x, y) f(y) d y, \\
\|g(x, \cdot)\|_{L^{n /(n-1)}(D)}=\sup _{\substack{f \in L^{n}(D) \\
\|f\|=1}} \int_{D} g(x, y) f(y) d y \leq C(\lambda, n, \operatorname{diam} D) .
\end{gathered}
$$

If the coefficients are not continuous, it is still possible to give a definition of a Green's function, as we will see in $\S I$, even though in this case uniqueness is known only in a few cases (see [5]).

In the study of such solutions a priori estimates on $u$ and its derivatives with constants independent of the regularity of the coefficients are particularly important because they may be carried through to the limit.

Evans [6] and Lin [9] showed respectively that the gradient and the second derivatives of a solution $u$ to the Dirichlet problem $(0.2)$ are integrable to a small power. Their results have in common the fact that they can be obtained from the property that if $g(x, y)$ is a Green's function for $L$, then $1 / g(x, \cdot)$ is integrable to some small power (see $\S I$ ). It is evident from their proofs that there is a strict relation between the integrability of the derivatives of $u$ and that of $1 / g$, in the sense that the better the latter one the better the first. Therefore a natural question that arises from these considerations is whether it is possible to improve the theorem on the integrability of $1 / \mathrm{g}$. In this paper we show that the answer is negative and that the Green's function can be quite singular. In fact, in general $1 / g$ is not globally integrable (as was shown by Trudinger in an unpublished result, which we state and prove in $\S I I)$, and in $\S \S I I I$ and IV we construct examples of operators for which $1 / \mathrm{g}$ is not even locally integrable. More precisely in $\S I I I$ we construct an example in the unit ball $B=B_{1}(0)$ in which 0 is a point of Lebesgue continuity for the coefficients of the operator $L$ but $1 / g$ is not integrable in any neighborhood of 0 . In $\S I V$ we construct an example of an operator $L$ in $B$ for which for all points $y_{0}$ in a set of positive measure $E \subset B, 1 / g(x, y)$ is not integrable in any neighborhood of $y_{0}$.

\section{Preliminary Results}

We are going to extend the concept of Green's function to the case in which the coefficients are not necessarily continuous, starting with the following definitions.

Definition 1. A regularization $\left\{a_{i j}^{k}(x)\right\}, i, j=1, \ldots, n$ and $k=1,2, \ldots, \infty$, of the coefficients of $L$, is a collection of smooth functions such that:

(i) for each pair $i j, a_{i j}^{k} \rightarrow a_{i j}$ a.e. in $D$ and uniformly on compact subsets of $D \backslash \bar{E}$, where $E$ is the set of points of discontinuity of $a_{i j}$;

(ii) for each $k,\left\{a_{i j}^{k}(x)\right\}$ satisfies $(0.1)$ with the same constants as $\left\{a_{i j}(x)\right\}$.

The operator $L^{k}=\sum_{i, j=1}^{n} a_{i j}^{k}(x) D_{i j}^{2}$ will be called a regularization of the operator $L$.

Observe that a regularization of $L$ can be obtained, for example, through convolution of the coefficients of $L$ with a smoothing kernel.

Suppose that the coefficients of $L$ are not continuous and consider some regularization $L^{k}$ of $L$. Let $L^{k} u^{k}=-f$ in $D, u^{k}=0$ on $\partial D$, and $g^{k}(x, y)$ 
be the corresponding Green's functions. Because of $(0.3)$ for every $x \in D$ there exists a subsequence, which we will still call $g^{k}$, such that $g^{k}(x, \cdot) \rightarrow$ $g(x, \cdot)$ weakly in $L^{n /(n-1)}(D)$. Through a diagonalization process we can define $g(x, y)$ for $x \in \mathbb{Q}^{n} \cap D$ (where $\mathbb{Q}^{n}$ is the subset of points of $\mathbb{R}^{n}$ with rational coordinates) and then, because of the equicontinuity of $\left\{u^{k}\right\}$, extend it to $D$. Observe that this process constructs a Green's function but says nothing about the uniqueness of $g$.

We are now going to recall the definition and some results about adjoint solutions.

Definition 2. An adjoint solution to an operator $L$ in $D$ is a function $v \in$ $L_{\text {loc }}^{1}(D)$ such that for every smooth domain $F$ with $\bar{F} \subseteq D, u \in C^{2}(\bar{F})$, $u=0$, and $|\nabla u|=0$ on $\partial F$, we have $\int_{F} v L u d x=0$.

The next theorem regards integrability properties of adjoint solutions; in particular, it states that nonnegative adjoint solutions belong to the Muckenhoupt class $A_{\infty}$ (for a definition see, e.g., [2]). This implies, in particular, integrability of small powers of reciprocals of Green's functions in domains that do not contain the pole.

Theorem 1.1. Let $v$ be a nonnegative adjoint solution to $L$ in $D$ and let $F$ be an open set such that $\bar{F} \subset D$. Then for some $p>0, p$ depending only on $\lambda$, $\Lambda$, and $n, v \in A_{p}(F)$.

The above theorem was proved for an operator with continuous coefficients by Bauman in [2] and shown to hold independently of the regularity of the coefficients by Fabes and Stroock [7].

Finally let us state Evans's and Lin's theorems.

Theorem 1.2 (Evans [6]). Let $v \geq 0$ be an adjoint solution to $L$ in a neighborhood of $B_{1}$ and $u \in C^{2}\left(\bar{B}_{1}\right), u=0$ on $\partial B_{1}$, and set $f=L u$. Then

(i)

$$
\int_{B_{1}} v|\nabla u(x)|^{2} d x \leq C\|f\|_{L^{n}\left(B_{1}\right)}^{2}\|v\|_{L^{n /(n-1)}\left(B_{1}\right)}
$$

(ii) there exists $\varepsilon=\varepsilon(\lambda, \Lambda)$ such that $\int_{B_{1}}|\nabla u|^{\varepsilon} d x \leq C\|f\|_{L^{n}\left(B_{1}\right)}^{2}$.

Theorem 1.3 (Lin [9]). Let $u$ be a solution to (0.2) in $B_{1}$ with $f \in L^{n}\left(B_{1}\right)$ and $\varphi=0$. Then there exists $\delta=\delta(\lambda, \Lambda)$ such that

$$
\int_{B_{1 / 2}}\left|D^{2} u\right|^{\delta} d x \leq C\|f\|_{L^{n}\left(B_{1}\right)}, \quad \text { where }\left|D^{2} u\right|=\left(\sum_{i, j=1}^{n}\left|D_{i j}^{2} u\right|^{2}\right)^{1 / 2} \text {. }
$$

\section{LACK OF INTEGRABILITY OF THE RECIPROCAL OF A GREEN'S FUNCTION: THE GLOBAL CASE}

By looking at the proofs of Theorems 1.2 and 1.3 we notice that if it were possible to prove that $\int_{B_{1}} \frac{1}{G(x, y)} d x<C(\lambda, \Lambda, n)<\infty$, we could prove $L^{1}$ estimates for the gradient of $u$ and improve the estimates for the second derivative of $u$. Unfortunately when the coefficients are not continuous the Green's function can be quite singular, as we will see with the following counterexamples.

In what follows $B_{r}=B_{r}(0)$, unless otherwise specified. 
Theorem 2.1 (Trudinger). There exist $0<\lambda<\Lambda$ and an elliptic operator $L=\sum_{i, j=1}^{n} b_{i j}(x) D_{i j}^{2}$ in $B_{1}$ with ellipticity parameters $\lambda$ and $\Lambda$ such that $1 / G_{L, B_{1}}(x, \cdot) \notin L^{1}\left(B_{r}\right)$, for $r<1$, where $G_{L, B_{1}}(x, y)$ is a Green's function for $L$ in $B_{1}$ and $x \notin \bar{B}_{r}$.

Proof. Observe that if $u$ is any $C^{2}$ function on $B_{1}$ (except possibly on a closed set of points of measure zero) and $H u=\left[D_{i j}^{2} u\right]_{i, j=1}^{n}$ is the Hessian matrix of $u$ with eigenvalues $C_{i}(x) \quad(i=1, \ldots, n)$, given any elliptic operator $L$ with ellipticity parameters $\lambda$ and $\Lambda$, then $L u=\sum_{i, j=1}^{n} a_{i j}(y) D_{i j}^{2} u=$ $\operatorname{trace}(A(y) H u(y))=\sum_{i=1}^{n} C_{i}(y) \alpha_{i}(y)$, where $A(y)=\left[a_{i j}(y)\right]_{i, j=1}^{n}$ is the matrix of the coefficients of $L$ and $\lambda \leq \alpha_{i}(y) \leq \Lambda$. Vice-versa, given $\lambda \leq \alpha_{i}(y) \leq \Lambda$, $L u=\sum_{i=1}^{n} \alpha_{i}(y) C_{i}(y)$ is an elliptic operator acting on $u$, with coefficients $a_{i j}(y)=\sum_{k=1}^{n} q_{i k}(y) \alpha_{k}(y) q_{j k}(y)$ (depending on $u$ ) and ellipticity parameters $\lambda$ and $\Lambda$.

Also, given $u$ and $L$, let $C_{i}^{+}(y)$ and $C_{j}^{-}(y)$ be respectively the nonnegative and the negative eigenvalues of $H u(y)$ and $L u=\sum_{i=1}^{n} \alpha_{i}(y) C_{i}(y)$. Now define the operator $\widetilde{L}$, in such a way that

$$
\tilde{L} u=\sum\left(\alpha_{i}(y)+1\right) C_{i}^{+}(y)+\sum \frac{\alpha_{j}(y)}{2} C_{j}^{-}(y) .
$$

$\widetilde{L}$ is clearly an elliptic operator with parameters $\lambda / 2$ and $\Lambda+1$.

Moreover we can write

$$
\widetilde{L} u=L u+\gamma(y)\left|D^{2} u(y)\right|,
$$

where $\left|D^{2} u(x)\right|=\left\{\sum_{i, j=1}^{n}\left|D_{i j}^{2} u(x)\right|^{2}\right\}^{1 / 2}$ and

$$
\gamma(y)=\left(\sum\left|C_{i}^{+}(y)\right|+\sum \frac{\alpha_{j}}{2}\left|C_{j}^{-}(y)\right|\right) /\left|D^{2} u(y)\right|
$$

satisfies $0<c(\lambda, \Lambda, n) \leq \gamma(y) \leq 1 / c(\lambda, \Lambda, n)$, for $y \in B_{1}$. Now consider the function $v(x)=|x|^{-s}-1$ for $|x| \leq 1$ and let $L_{v}$ be the operator defined by $L_{v} v=\Lambda \sum C_{j}^{-}+\lambda \sum C_{i}^{+}$for some $0<\lambda \leq \Lambda$ and where $C_{i}^{+}(y)$ and $C_{j}^{-}(y)$ are the eigenvalues of $H v(y)$. Since $D_{i j}^{2} v(x)=-s|x|^{-s-2}\left\{\delta_{i j}-(s+2) x_{i} x_{j} /|x|^{2}\right\}$, it is easy to see that $H v(x)$ has eigenvalues $-s|x|^{-s-2}$ with multiplicity $(n-1)$ and $s(s+1)|x|^{-s-2}$ with multiplicity 1 , so that if we choose $s \leq[(n-1) \Lambda / \lambda]-1$, then $L_{v} v=-s|x|^{-s-2}\{(n-1) \Lambda-(s+1) \lambda\} \leq 0$ for $x \neq 0$.

Now let $u=-v$ and let $\widetilde{L}_{v}$ be the operator associated to $L_{v}$ and $u$ in the way described in (2.2) and (2.3). We will show that $\widetilde{L}_{v}$ satisfies the hypothesis for the operator $L$ in the statement of the theorem. From now on let $L=\widetilde{L}_{v}$ and $G_{L, B_{1}}(x, y)$ be a Green's function for this operator in $B_{1}$, as defined before.

Define

$$
u_{\varepsilon}(x)= \begin{cases}u, & |x|>\varepsilon, \\ a_{\varepsilon}|x|^{2}+b_{\varepsilon}, & |x| \leq \varepsilon,\end{cases}
$$

where $a_{\varepsilon}$ and $b_{\varepsilon}$ are chosen in such a way that $a_{\varepsilon} r^{2}+\left.b_{\varepsilon}\right|_{r=\varepsilon}=\bar{u}(\varepsilon)$ and $\left.\frac{d}{d r}\left[a_{\varepsilon} r^{2}+b_{\varepsilon}\right]\right|_{r=\varepsilon}=\left.\frac{d}{d r}[\bar{u}(r)]\right|_{r=\varepsilon}$. (In particular, $a_{\varepsilon}=s \varepsilon^{-s-2} / 2>0$.) 
Observe $u_{\varepsilon} \in C^{1,1}\left(B_{1}\right) \cap L^{\infty}\left(B_{1}\right)$ has bounded weak second derivatives, and letting $A(x)$ be the matrix of the coefficients of $L$

$$
L u_{\varepsilon}=\left\{\begin{array}{ll}
L u, & |x|>\varepsilon, \\
2 a_{\varepsilon} \operatorname{tr} A(x), & |x| \leq \varepsilon,
\end{array} \text { and } 2 a_{\varepsilon} \operatorname{tr} A(x)>0\right.
$$

Choose $x_{0}$ with $\left|x_{0}\right|>\varepsilon$. Then recalling the definition of $L$

$$
\begin{aligned}
v\left(x_{0}\right) & =-u\left(x_{0}\right)=\int_{B_{1}} G_{L, B_{1}}\left(x_{0}, y\right) L u_{\varepsilon}(y) d y \\
& =\int_{|y| \leq \varepsilon} G_{L, B_{1}}\left(x_{0}, y\right) 2 a_{\varepsilon} \operatorname{tr} A(y) d y+\int_{\varepsilon<|y| \leq 1} G_{L, B_{1}}\left(x_{0}, y\right) L u(y) d y \\
& >\int_{\varepsilon<|y| \leq 1} G_{L, B_{1}}\left(x_{0}, y\right) L_{v} u(y) d y+\int_{\varepsilon<|y| \leq 1} G_{L, B_{1}}\left(x_{0}, y\right) \gamma(y)\left|D^{2} u(y)\right| d x \\
& >C \int_{\varepsilon<|y| \leq 1} G_{L, B_{1}}\left(x_{0}, y\right)\left|D^{2} u(y)\right| d y .
\end{aligned}
$$

In other words $\int_{\varepsilon<|y| \leq 1} G_{L, B_{1}}\left(x_{0}, y\right)\left|D^{2} u(y)\right| d y<C v\left(x_{0}\right)$.

If $\int_{B_{r}} \frac{1}{G_{L, B_{1}}\left(x_{0}, y\right)} d y<C$ for some $r<1$, then from Theorem 1.2 it follows that for $\varepsilon<r$,

$$
\begin{aligned}
\int_{r \geq|y|>\varepsilon}\left|D^{2} u(y)\right|^{1 / 2} d y \leq & \left\{\int_{r \geq|y|>\varepsilon} G_{L, B_{1}}\left(x_{0}, y\right)\left|D^{2} u(y)\right| d y\right\}^{1 / 2} \\
& \times\left\{\int_{r \geq|y|>\varepsilon} \frac{1}{G_{L, B_{1}}\left(x_{0}, y\right)} d y\right\}^{1 / 2} \leq C v\left(x_{0}\right)^{1 / 2},
\end{aligned}
$$

which is clearly impossible for $s>2 n-2$ since $\left|D^{2} u(x)\right| \simeq C|x|^{-s-2}$. So the theorem is proved if we choose $2 n-2<s \leq(n-1) \Lambda / \lambda$ and appropriate $\Lambda$ and $\lambda$.

Corollary 2.3. For the operator $L$ in Theorem 2.1 we also have that there exists a $p<1$ such that $\left(1 / G_{L, B_{1}}\left(x_{0}, \cdot\right)\right)^{p} \notin L^{1}\left(B_{r}\right)$, for every $r<1$.

Proof. Since $s>2 n-2$, there exists $q>2$ such that $s>q n-2$. Let $p=1 /(q-1)$; if $\int_{B_{r}}\left(1 / G_{L, B_{1}}\left(x_{0}, \cdot\right)\right)^{p} d y<C$ we would have

$$
\begin{aligned}
\int_{r \geq|y|>\varepsilon}\left|D^{2} u(y)\right|^{1 / q} d y \leq & \left\{\int_{r \geq|y|>\varepsilon} G_{L, B_{1}}\left(x_{0}, y\right)\left|D^{2} u(y)\right| d y\right\}^{1 / q} \\
& \times\left\{\int_{r \geq|y|>\varepsilon}\left(\frac{1}{G_{L, B_{1}}\left(x_{0}, y\right)}\right)^{p} d y\right\}^{1-1 / q} \\
\leq & C v\left(x_{0}\right)^{1 / q} .
\end{aligned}
$$

But because of the choice of $q$, we have $(s+2) / q>n$ and therefore $\left|D^{2} u(x)\right|^{1 / q}$ $\simeq C|x|^{-(s+2) / q}$ is not integrable on $B_{\varepsilon}$ as $\varepsilon \rightarrow 0$. 


\section{LACK OF INTEGRABILITY OF THE RECIPROCAL OF A GREEN'S FUNCTION: THE LOCAL CASE}

We may wonder if a weaker kind of estimate holds, such as that for almost every $x \in D$, there exists $r>0$ such that $|\nabla u| \in L^{1}\left(B_{r}(X)\right)$. As before, if we could prove that

(a) for a.e. $x \in D$, there exists $r>0$ such that $1 / G_{L, B_{1}}\left(x_{0}, \cdot\right) \in L^{1}\left(B_{r}(x)\right)$, with $x_{0} \notin \bar{B}_{r}(x)$,

then the above property would follow again from the proof of Theorem 1.2.

Again we will see in Theorems 3.2 and 3.3 that property (a) for a Green's function does not generally hold. In Theorem 3.3 we will construct an example of an operator $L$ in $B_{1}$ with Green's function $g$ for which the set $E=\{z \in$ $B_{1} \mid 1 / g(x, \cdot) \notin L^{1}\left(B_{r}(z)\right) \forall r$ and $\left.x \notin \bar{B}_{r}\right\}$ has positive measure.

We first prove, in Theorem 3.2, a particular case of Theorem 3.3, precisely that Lebesgue continuity points for the coefficients of $L$ may be in $E$. We should point out the reasons why we think it is important to state and prove this theorem separately. First of all, when we first became interested in property (a) we had just learned of some regularity results proved by Caffarelli, namely, "weak $C^{1, \alpha}$ " estimates for solutions in a neighborhood of a point of Lebesgue continuity for the coefficients of $L$ (see [4]). Therefore we thought that if property (a) held there was a good likelihood it held at those points. Moreover we think this simpler case may clarify better the technique used to construct both counterexamples.

We will need the following lemma.

Lemma 3.1. A sufficient condition for $\int_{\Omega}[f(x)]^{-p} d x<\infty$ for all $p<1$ is that there exists a constant $c$ such that for every measurable $E \subset \Omega, \int_{E} f(x) d x \geq$ $c|E|^{2}$. Moreover if $\int_{\Omega}[f(x)]^{-1} d x<\infty$, then $\int_{E} f(x) d x \geq c|E|^{2}$, for some constant $c$ independent of $E$.

Proof. To prove the first statement let $E_{\lambda}=\{x \in \Omega \mid f(x) \leq \lambda\}$ and let $\widetilde{E}_{\lambda}=$ $\{x \in \Omega \mid 1 / f(x) \geq \lambda\}$. Then $\lambda\left|E_{\lambda}\right| \geq \int_{E_{\lambda}} f(x) d x \geq c\left|E_{\lambda}\right|^{2}$, which means $\left|E_{\lambda}\right| \leq$ $C \lambda$. Therefore, $\left|\widetilde{E}_{\lambda}\right| \leq C / \lambda$, which implies

$$
\begin{aligned}
\int_{\Omega} \frac{1}{f(x)^{p}} d x & =\int_{\Omega \backslash \widetilde{E}_{1}} \frac{1}{f(x)^{p}} d x+p \int_{1}^{\infty} \lambda^{p-1}\left|\widetilde{E}_{\lambda}\right| d \lambda \\
& \leq|\Omega|+p C \int_{1}^{\infty} \lambda^{p-2} d \lambda<\infty \quad \text { for all } p<1
\end{aligned}
$$

For the second part of the theorem, observe that

$$
\begin{aligned}
|E| & =\int_{E} f(x)^{1 / 2} \frac{1}{f(x)^{1 / 2}} d x \\
& \leq\left\{\int_{E} f(x) d x\right\}^{1 / 2}\left\{\int_{E} \frac{1}{f(x)} d x\right\}^{1 / 2} \leq c\left\{\int_{E} f(x) d x\right\}^{1 / 2} .
\end{aligned}
$$

Theorem 3.2. There exists an elliptic operator $L=\sum_{i, j=1}^{n} a_{i j}(x) D_{i j}^{2}$ defined on the unit ball $B_{1}=B_{1}(0)$, with coefficients $a_{i j}$ bounded and measurable on $B_{1}$, and Lebesgue continuous at the origin such that for any ball $B_{r}=B_{r}(0)$ with $r<1,1 / G_{L, B_{1}}(x, \cdot) \notin L^{1}\left(B_{r}\right)$, where $G_{L, B_{1}}(x, y)$ is a Green's function for $L$ in $B_{1}$ and $x \notin \bar{B}_{r}$. 
Proof. For each $B_{r}\left(x_{0}\right)$ let $L_{r, x_{0}}=\sum_{i, j=1}^{n} a_{i j}\left[\left(x-x_{0}\right) / r\right] D_{i j}^{2}$ where the $a_{i j}$ are the coefficients for $L$ in Trudinger's operator and $x \in B_{r}\left(x_{0}\right)$. Then clearly if $G_{L_{r, x_{0}}}(x, y)$ is a Green's function for $L_{r, x_{0}}$ in $B_{r}\left(x_{0}\right), 1 / G_{L_{r, x_{0}}}(x, \cdot) \notin$ $L^{1}\left(B_{r^{\prime}}\left(x_{0}\right)\right)$, for $r^{\prime} \leq r$ where $x \notin \bar{B}_{r^{\prime}}\left(x_{0}\right)$.

To actually prove the theorem, let $\left\{x_{k}\right\}$ be a sequence of points such that $\left|x_{k}\right|=1 / k$ for $k=2,3, \ldots$, let $r_{k}=1 / 5 k^{2}$, and set $B_{k}=B_{r_{k}}\left(x_{k}\right)$.

Then define $L$ as follows:

$$
L= \begin{cases}L_{r_{k}, x_{k}} & \text { in } B_{k}, \\ \Delta & \text { in } B_{1} \backslash \bigcup_{k=2}^{\infty} B_{k}\end{cases}
$$

where $L_{r_{k}, x_{k}}$ are the operators constructed above and $\Delta$ is the Laplace's operator.

We claim that $L$ satisfies the theorem. First of all its coefficients are Lebesgue continuous at the origin. To see this, let $a_{i j}$ indicate the coefficients of $L$ and $a_{i j}^{k}$ the coefficients of $L_{r_{k}, x_{k}}=L_{k}$. Then $a_{i j}(0)=\delta_{i j}$ and for any $r>0$,

$$
\begin{aligned}
\int_{B_{r}}\left|a_{i j}(x)-\delta_{i j}\right|^{n} d x & \leq \int_{\bigcup_{k>1 / r-1} B_{k}}\left|a_{i j}^{k}(x)-\delta_{i j}\right|^{n} d x \\
& \leq C(\Lambda, n) \sum_{k>1 / r-1}\left|B_{k}\right| \leq C(\Lambda, n) \frac{r^{n}}{(1-r)^{n}} \sum_{k>1 / r-1} \frac{1}{k^{n}} .
\end{aligned}
$$

Therefore

$$
\frac{1}{\left|B_{r}\right|} \int_{B_{r}}\left|a_{i j}(x)-\delta_{i j}\right|^{n} d x \leq C(\Lambda, n) \frac{1}{(1-r)^{n}} \sum_{k>1 / r-1} \frac{1}{k^{n}},
$$

which tends to zero with $r$.

We now need to prove that letting $G_{L, B_{1}}(x, y)$ be a Green's function for $L$ in $B_{1}$, we have $1 / G_{L, B_{1}}(x, \cdot) \notin L^{1}\left(B_{r}\right)$ for $r<1, B_{r}=B_{r}(0)$, and $x \notin \bar{B}_{r^{\prime}}$.

We will use the following notation: if $B=B_{r}\left(x_{0}\right)$, then $B^{s}=B_{s r}\left(x_{0}\right)$.

Fix $r>0$; then for some $k, B_{k} \subset B_{r}$. Let $L^{j}$ and $L_{k}^{j}$ be the operator obtained by regularizing the coefficients of $L$ and $L_{k}$ respectively, with a kernel supported in the ball of radius $1 / 5 j$. Moreover let $G^{j}(x, y)$ and $G_{k}^{j}(x, y)$ be the Green's functions for $L^{j}$ in $B_{1}$ and for $L_{k}^{j}$ in $B_{k}$.

Let $x_{0} \in B_{1} \backslash \bar{B}_{k}$ and $x_{1} \in B_{k}^{3 / 4} \backslash \bar{B}_{k}^{1 / 2}$. We can assume, without loss of generality, that $G^{j}\left(x_{0}, y\right) \rightarrow G_{L, B_{1}}\left(x_{0}, y\right)$ and $G_{k}^{j}\left(x_{1}, y\right) \rightarrow G_{k}\left(x_{1}, y\right)$ weakly in $L^{n /(n-1)}\left(B_{k}\right)$. For $j>2 k$ the coefficients of $L^{j}$ and of $L_{k}^{j}$ coincide in $B_{k}^{1 / 2}$.

Assume the following claim (to be proved later).

Claim. There exists a subsequence of $\left\{G^{j}\left(x_{0}, y\right)\right\}$ and a subsequence of $\left\{G_{k}^{j}\left(x_{1}, y\right)\right\}$, such that $G_{k}^{j}\left(x_{1}, y\right) / G^{j}\left(x_{0}, y\right)>C$ for all $y \in B_{k}^{1 / 8}$, with $C$ depending at most on $\lambda, \Lambda, n$, and $r_{k}$.

We will show that it implies the theorem.

Call the two subsequences again $\left\{G^{j}\right\}$ and $\left\{G_{k}^{j}\right\}$ and assume that

$$
\int_{B_{r}} \frac{d y}{G_{L, B_{1}}\left(x_{0}, y\right)}<C \text {. }
$$


Then clearly also $\int_{B_{k}^{1 / 8}}\left[G_{L, B_{1}}\left(x_{0}, y\right)\right]^{-1} d y<C$, and for measurable $E \subset B_{k}^{1 / 8}$ Lemma 3.1 implies that $\int_{E} G_{L, B_{1}}\left(x_{0}, y\right) d y \geq C|E|^{2}$; then

$$
\begin{aligned}
\int_{E} G_{k}\left(x_{1}, y\right) d y & =\lim _{j \rightarrow \infty} \int_{E} G_{k}^{j}\left(x_{1}, y\right) d y=\lim _{j \rightarrow \infty} \int_{E} \frac{G_{k}^{j}\left(x_{1}, y\right)}{G^{j}\left(x_{0}, y\right)} G^{j}\left(x_{0}, y\right) d y \\
& \geq C \lim _{j \rightarrow \infty} \int_{E} G^{j}\left(x_{0}, y\right) d y=C \int_{E} G_{L, B_{1}}\left(x_{0}, y\right) d y \geq C|E|^{2}
\end{aligned}
$$

and that then $\int_{B_{k}^{1 / 8}}\left[G_{k}(x, y)\right]^{-1} d y<C$ for all $p<1$, which was shown to be false in Corollary 2.4.

Finally let us prove the claim. Let $\bar{v}_{j}(y)=G_{k}^{j}\left(x_{1}, y\right) / G^{j}\left(x_{0}, y\right)$ in $B_{k}^{1 / 2}$; this is a quotient of adjoint solutions defined in [2] as a normalized adjoint solution by Bauman who also shows that a Harnack's inequality holds for $\bar{v}_{j}$ in $B_{k}^{1 / 8}$. Also, by Theorem 1.1 both $G^{j}\left(x_{0}, y\right)$ and $G_{k}^{j}\left(x_{1}, y\right)$ are $A^{\infty}$ weights as functions of $y$ in $B_{k}^{1 / 2}$ and, therefore, for a fixed $\varepsilon>0$ we can find $\alpha=$ $\alpha(\lambda, \Lambda, n, k)$ such that $\left|E_{j}\right| \geq(1-\varepsilon)\left|B_{k}^{1 / 2}\right|$ where

$$
\begin{aligned}
E_{j}= & \left\{z \in B_{k}^{1 / 2} \mid \alpha \int_{B_{k}^{1 / 2}} G^{j}\left(x_{0}, y\right) d y \leq G^{j}\left(x_{0}, z\right) \leq \frac{1}{\alpha} \int_{B_{k}^{1 / 2}} G^{j}\left(x_{0}, y\right) d y\right\} \\
& \cup\left\{z \in B_{k}^{1 / 2} \mid \alpha \int_{B_{k}^{1 / 2}} G_{k}^{j}\left(x_{1}, y\right) d y \leq G_{k}^{j}\left(x_{1}, z\right) \leq \frac{1}{\alpha} \int_{B_{k}^{1 / 2}} G_{k}^{j}\left(x_{1}, y\right) d y\right\} .
\end{aligned}
$$

Now let $E=\bigcap_{i=1}^{\infty} \bigcup_{j=i}^{\infty} E_{j}$ be the lim sup of the sets $E_{j}$ 's. Then also $|E| \geq$ $(1-\varepsilon)\left|B_{k}^{1 / 2}\right|$. By taking $\varepsilon$ small enough this guarantees that there exists $z_{0} \in$ $B_{k}^{1 / 8}$, which belongs to $E$ and therefore to infinitely many $E_{j}$. In other words there exists a subsequence of $\left\{G^{j}\right\}$ (which will be called again $G^{j}$ ) such that $G^{j}\left(x_{0}, z_{0}\right) \sim \int_{B_{k}^{1 / 2}} G^{j}\left(x_{0}, y\right) d y$ and the corresponding $G_{k}^{j}\left(x_{1}, z_{0}\right) \sim$ $\int_{B_{k}^{1 / 2}} G_{k}^{j}\left(x_{1}, y\right) d y$. Because of Harnack's inequality for normalized adjoint solutions (see [2]), we can replace $z_{0}$ with any $y \in B_{k}^{1 / 8}$ and get

$$
\frac{G_{k}^{j}\left(x_{1}, y\right)}{G^{j}\left(x_{0}, y\right)} \geq C \frac{\int_{B_{k}^{1 / 2}} G_{k}^{j}\left(x_{1}, y\right) d y}{\int_{B_{k}^{1 / 2}} G^{j}\left(x_{0}, y\right) d y} .
$$

Moreover observe that $\int_{B_{k}^{1 / 2}} G^{j}\left(x_{0}, y\right) d y \leq C\left\|G^{j}\left(x_{0}, \cdot\right)\right\|_{L^{n /(n-1)}\left(B_{k}^{1 / 2}\right)} \leq C$; by the doubling condition and the maximum principle $\int_{B_{k}^{1 / 2}} G_{k}^{j}\left(x_{1}, y\right) d y \geq$ $C(\Lambda, n) r_{k}^{2}$ for $x \in B_{k}^{3 / 4}$ and, in particular, for $x_{0}$.

The proof is now complete.

Theorem 3.3. There exists an elliptic operator $L=\sum_{i, j=1}^{n} a_{i j}(x) D_{i j}^{2}$ defined on the unit ball $B_{1}=B_{1}(0)$, with coefficients $a_{i j}$ bounded and measurable on $B_{1}$, for which a Green's function $G_{L, B_{1}}(x, y)$ exists, with the property, if $E=\left\{z \in B_{1} \mid 1 / G_{L, B_{1}}(x, \cdot) \notin L^{1}\left(B_{r}(z)\right) \forall r<d\left(z, \partial B_{1}\right)\right.$ and $\left.x \notin \bar{B}_{r}\right\}$, then $|E|>0$. 
Before proving the theorem we are going to need the following.

Lemma 3.4. For every $\varepsilon>0$, there exists a sequence of open cubes $\left\{Q_{j}\right\}_{j=1}^{\infty} \subset B_{1}$ such that

(i) $Q_{i} \cap Q_{j}=\varnothing$ for $i \neq j$;

(ii) $\bigcup_{j=1}^{\infty} Q_{j}$ is dense in $B_{1}$;

(iii) $\sum_{j=1}^{\infty}\left|Q_{j}\right| \leq \varepsilon$;

(iv) the centers of the $Q_{j}$ 's are dense in $B_{1} \backslash \bigcup_{j=1}^{\infty} Q_{j}$.

Proof. Consider the set $R$ of points of $B_{1}$ with rational coordinates; then $R \subset B_{1}, R$ is dense in $B_{1}$, and $R$ is countable. Let $\left\{x_{j}\right\}_{i=1}^{\infty}$ be an enumeration of the elements of $R$. For fixed $\varepsilon$, let

$$
r_{j}=\left(\varepsilon / \omega_{n}\right)^{1 / n} \min \left(1 / 2^{j}, d\left(x_{j}, \partial B_{1}\right)\right),
$$

where $\omega_{n}$ is the volume of the $n$-dimensional unit ball, and let $B_{j}=B_{r_{j}}\left(x_{j}\right)$. Then clearly $\bigcup_{j=1}^{\infty} B_{j}$ is dense in $B_{1}$ and

$$
\left|\bigcup_{j=1}^{\infty} B_{j}\right| \leq \sum_{j=1}^{\infty}\left|B_{j}\right| \leq \omega_{n} \frac{\varepsilon}{\omega_{n}} \sum_{j=1}^{\infty}\left(\frac{1}{2^{n}}\right)^{j} \leq \varepsilon .
$$

Let $A=\bigcup_{j=1}^{\infty} B_{j}$. Then $A$ is open and can therefore be expressed as a countable union of nonoverlapping cubes. These cubes satisfy (ii) and (iii).

To prove (iv), let $x \in B_{1} \backslash \bigcup_{j=1}^{\infty} Q_{j}$ and let us assume that there exists an open ball around $x, B_{r}=B_{r}(x)$, such that no one of the centers of the $Q_{j}$ 's belongs to $B_{r}$. Consider then $B_{r / 2}$; if there are only finitely many $Q_{j}$ 's that intersect $B_{r / 2}$ then since $x \notin Q_{j}$ for every $j$, we can find a neighborhood of $x$ that does not intersect any $Q_{j}$, violating the density of $\bigcup_{j=1}^{\infty} Q_{j}$. If infinitely many $Q_{j}$ intersect $B_{r / 2}$ then since all the centers are outside $B_{r}$, all of these cubes will have sides $>r c_{n} / 2$ with $c_{n}$ a constant depending on dimension. But this last fact violates (iii).

Proof of Theorem 3.3. In order to construct the operator $L$ let $B_{j}$ be the open balls inscribed in the cubes $Q_{j}$ in the above lemma. Then let

$$
L= \begin{cases}L_{j} & \text { in } B_{j}, \\ \Delta & \text { in } B_{1} \backslash \bigcup_{j=2}^{\infty} B_{j}\end{cases}
$$

where $L_{j}$ is the operator associated to the ball $B_{j}$ in the way described at the beginning of the proof of Theorem 2.4. Set $C=B_{1} \backslash \bigcup_{j=1}^{\infty} Q_{j}$.

We claim that $C \subset E$.

To see this let $z \in C$ and $B_{r}(z)$ be a ball centered at $z$ with radius $r<$ $d\left(z, \partial B_{1}\right)$. Then by (iv) of the lemma, there exists $z_{j}$, center of some $B_{j}$, with $z_{j} \in B_{r}(z)$. Then letting $G(x, y)=G_{L, B_{1}}(x, y)$ be a Green's function for $L$ in $B_{1}$ and $G_{j}(x, y)$ a Green's function for $L_{j}$ in $B_{j}$ by an argument analogous to the one in the proof of Theorem 2.4 , one shows that $1 / G_{L, B_{1}}\left(x,{ }^{\circ}\right)$ is not integrable over $B_{r}(z)$.

The author wishes to thank Professor Eugene B. Fabes for many helpful discussions about the contents of this paper. 


\section{REFERENCES}

1. A. D. Aleksandrov, Uniqueness conditions and bounds for the solution of the Dirichlet problem, Vestnik Leningrad. Univ. Math. 18 (1963), 5-29.

2. P. Bauman, Positive solutions of elliptic equations in nondivergence form and their adjoints, Ark. Mat. 22 (1984), 153-173.

3. __ A Wiener test for nondivergence structure, second-order elliptic equations, Indiana Univ. Math. J. 4 (1985), 825-844.

4. L. Caffarelli, Elliptic second order equations, Rend. Sem. Mat. Fis. Milano 57 (1988).

5. M. C. Cerutti, L. Escauriaza, and E. B. Fabes, Uniqueness for the Dirichlet problem for some elliptic operators with discontinuous coefficients, Ann. Mat. Pura Appl. (to appear).

6. C. Evans, Some estimates for nondivergence structure second order elliptic equations, Trans. Amer. Math. Soc. 287 (1985), 701-712.

7. E. Fabes and D. Strook, The $L^{p}$-integrability of Green's functions and fundamental solutions for elliptic and parabolic equations, Duke Math. J. 51 (1984), 977-1016.

8. D. Gilbarg and N. S. Trudinger, Elliptic partial differential equations of second order, Springer-Verlag, New York and Berlin, 1983.

9. F.-H. Lin, Second derivative $L^{p}$-estimates for elliptic equations of nondivergence form, Proc. Amer. Math. Soc. 96 (1986), 447-451.

10. B. Oksendhal, Dirichlet forms, quasiregular functions and Brownian motion, Invent. Math. 91 (1988), 273-297.

11. C. Pucci, Limitazioni per soluzioni di equazioni ellittiche, Ann. Mat. Pura Appl. 74 (1966), 15-30.

12. M. V. Safanov, Harnack's inequality for elliptic equations and the Hölder property of their solutions, Zap. Nauchn. Sem. Leningrad. Odtel. Mat. Inst. Steklov. (LOMI) 96 (1980), 272-287; English transl. in J. Soviet Math. 21 (1983).

Dipartimento di Matematica, Politecnico di Milano, Piazza leonardo da Vinci 32 20133 Milano, Italy

E-mail address: cricer@ipmma1.polimi.it 